\title{
Characterization of electron microscopes with binary pseudo-random multilayer test samples*
}

\author{
Valeriy V. Yashchuk ${ }^{1}$, Raymond Conley ${ }^{2}$, Erik H. Anderson ${ }^{3}$, Samuel K. Barber ${ }^{1}$, \\ Nathalie Bouet ${ }^{2}$, Wayne R. McKinney ${ }^{1}$, Peter Z. Takacs ${ }^{4}$, Dmitriy L. Voronov ${ }^{1}$ \\ ${ }^{1}$ Advanced Light Source, Lawrence Berkeley National Laboratory, Berkeley, CA 94720, USA \\ ${ }^{2}$ NSLS-II, Brookhaven National Laboratory, Upton, NY 11973, USA \\ ${ }^{3}$ Center for X-ray Optics, Lawrence Berkeley National Laboratory, Berkeley, CA 94720, USA \\ ${ }^{4}$ Brookhaven National Laboratory, Upton, NY 11973, USA
}

* The Advanced Light Source is supported by the Director, Office of Science, Office of Basic Energy Sciences, Material Science Division, of the U.S. Department of Energy under Contract No. DE-AC0205CH11231 at Lawrence Berkeley National Laboratory. Research at Brookhaven National Laboratory is sponsored by the U.S. Department of Energy under Contract No. DE-AC02-98CH10886.

\begin{abstract}
:
Verification of the reliability of metrology data from high quality x-ray optics requires adequate methods for test and calibration of the instruments be developed. For such verification of optical surface profilometers in the spatial frequency domain, a modulation transfer function (MTF) calibration method based on binary pseudo-random (BPR) gratings and arrays has been suggested [1-3] and proven to be an effective calibration method for a number of interferometric microscopes, a phase shifting Fizeau interferometer, and a scatterometer [4].

Here we describe the details of development of binary pseudo-random multilayer (BPRML) test samples suitable for characterization of scanning (SEM) and transmission (TEM) electron microscopes. A BPRML sample is a multilayer structure consisting of two materials with significantly different contrasts when observed with an electron microscope. The thicknesses of the layers are distributed according to a binary pseudo-random sequence. For the MTF calibration of an electron microscope, an image of the BPR multilayer cross-section, measured with the microscope, is processed to get a power spectral density (PSD) distribution. The MTF is found as the square root of the ratio of the PSD spectrum measured with the BPRML to the 'ideal', spatial frequency independent, PSD spectrum, inherent for a precisely fabricated BPRML.

We discuss the results of SEM and TEM measurements with the BPRML test samples fabricated from a BPRML (WSi $2 / \mathrm{Si}$ with fundamental layer thickness of $3 \mathrm{~nm}$ ) with a Dual Beam FIB (focused ion beam)/SEM technique. In particular, we demonstrate that significant information about the metrological reliability of the TEM measurements can be extracted even when the fundamental frequency of the BPRML sample is smaller than the Nyquist frequency of the measurements. The measurements demonstrate a number of problems related to the interpretation of the SEM and TEM data. Note that similar BPRML test samples can be used to characterize x-ray microscopes. Corresponding work with x-ray microscopes is in progress.
\end{abstract}

\section{References:}

[1] V.V. Yashchuk, W.R. McKinney, and P.Z. Takacs, Opt. Eng. 47(7), 073602-1-5 (2008).

[2] S.K. Barber, P. Soldate, E.D. Anderson, R. Cambie, W.R. McKinney, P.Z. Takacs, D.L. Voronov, and V.V. Yashchuk, J. Vac. Sci. and Tech. B 27(6), 3213-9 (2009).

[3] S.K. Barber, E.H. Anderson, R. Cambie, S. Marchesini, W.R. McKinney, P.Z. Takacs, D.L. Voronov, V.V. Yashchuk, Opt. Eng. 49(5), 053606/1-9 (2010).

[4] S.K. Barber, E.D. Anderson, R. Cambie, W.R. McKinney, P.Z. Takacs, J.C. Stover, D.L. Voronov, V.V. Yashchuk, Nucl. Instr. and Meth. A 616, 172-182 (2010).

Ext. Abstract to The $16^{\text {th }}$ Pan-American Synchrotron Radiation Instrumentation Conference (September 21-24, 2010, Argonne National Laboratory, USA). 